\title{
Anaesthesia and cancer recurrence
}

Volume I Issue 3 - 2014

\begin{abstract}
Abbreviations: NK Cells, Natural Killer Cells; CTL, Cytotoxic Lymphocytes; STAT, Signal Transducer and Activator of Transcription; SNS, Sympathetic Nervous System; VEGF, Vascular Endothelial Growth Factor; HIF-1 $\alpha$, Hypoxia Inducible Factor-1 $\alpha$; VGSC, Voltage Gated Sodium Channels; HPA, HypothalamicPituitary Adrenal Axis
\end{abstract}

\section{Editorial}

The incidence of cancer is increasing because of improved screening practices. Surgical resection of a tumor offers the best prospect for a good outcome in such patients. However, the risk of minimum residual disease and tumor seeding seems inevitable even after a carefully conducted procedure with wide margins of normal tissue resected. ${ }^{1,2}$

Recent clinical data have pointed towards a potentially devastating consequence of exposure to anaesthetic agents on cancer recurrence and metastasis. ${ }^{3-7}$ During surgery bacterial contamination is inevitable but all the patients do not land up in sepsis. Similarly, inadvertent seeding of tumor cells during surgical procedure eventually leading to clinical metastasis is a complex dynamic process and depends on the delicate balance between host immune surveillance and tumor survival. The process of metastasis can be divided into four steps namely epithelial-mesenchymal transition, intravasation into blood vessels, migration of viable prometastatic cells (only $0.01 \%$ cells survive) and finally proliferation of these metastatic progenitor cells. ${ }^{8-10}$ Survival of circulating tumor cells in circulation depends upon host immunity and inflammatory mediators released by immune cells in response. The surgical stress response, general anaesthesia with inhalation agents and opioids usage (especially morphine) in the vulnerable perioperative period leads to immunosupression which has been implicated in promoting tumor recurrence. ${ }^{5,6,11}$ Natural killer (NK) cells play a dominant role in eliminating cancer cells along with T helper 1 (Th1) cells and cytotoxic lymphocytes (CTL). ${ }^{12,13}$ Proinflammatory cytokines, catecholamine and signal transducer and activator of transcription 3 (STAT 3) are implicated in cancer metastasis and recurrence. Activation of sympathetic nervous system (SNS) and hypothalamic-pituitary adrenal axis (HPA) plays a central role in immune suppression resulting from surgical stress. Prostaglandins secreted from tumour cells and catecholamines suppress NK cells, Th1 cells and CTL. They also shift the Th1/Th2 balance toward Th2 type immune response and promote angiogenesis. ${ }^{11-13}$ Glucocorticoids increase apoptosis of immature $\mathrm{T}$ cells in thymus.

Along with surgical stress a number of perioperative factors like blood transfusion, ${ }^{14}$ hypothermia, ${ }^{15}$ hyperglycaemia, ${ }^{16}$ hypotension, ${ }^{17}$ hypoxia and postoperative pain $^{18}$ also suppress host immunity. Patients with malignancy undergoing major surgery are often anaemic and may often require perioperative blood transfusions. Intra-operative blood transfusion increases the risk of cancer recurrence by $74 \% .^{19}$ This is due to the well known phenomenon of transfusion related immunomodulation (TRIM). ${ }^{14}$ Thus, for cancer patients undergoing major surgery, optimising the nutritional status and use of erythropoietin to build up haemoglobin, may be

\author{
Rakesh Garg,' Anju Gupta² \\ 'Department of Anesthesiology, Pain and Palliative Care,All India \\ Institute of Medical Sciences, India \\ ${ }^{2}$ Department of Anesthesiology, Delhi State Cancer Institute, \\ India
}

Correspondence: Rakesh Garg, Department of Anaesthesiology, Pain and Palliative Care, All India Institute of Medical Sciences, Ansari Nagar, New Delhi- I I0029, India, Tel 91-98I0394950, Email drrgarg@hotmail.com

Received: July 21, 2014 | Published: July 22, 2014

considered preoperatively (if feasible) to avoid/ reduce need for blood transfusion. But this may not be possible in all the cases due to urgent need of the cancer surgery. We must take measures to minimise blood loss like optimal surgical technique, maintain normothermia, use anfibrinolytic, and maintain the volume status with appropriate fluids till the transfusion threshold is reached. Stored blood and old erythrocytes release pro-tumour mediators. Leucodepleted blood may offer some protection but their use remains controversial. ${ }^{20}$

Hypotension activates SNS and HPA with its consequent immunosuppressive effects. ${ }^{17}$ Hypoxia leads to Th-1 suppression and activates hypoxia- inducible factors in immune cells as well as cancer cells. ${ }^{18} \mathrm{HIF}$ inhibit NK cells and induces pro-angiogenic factors in tumour cell including VEGF and angiopoietin2. Number of hypotensive episodes during surgery found to be significant risk factor for recurrence after liver resection for metastatic colorectal cancer. Hypothermia also suppresses NK cell activity and increased risk of tumour progression, due possibly to SNS activation..$^{15}$ Hyperglycemia suppresses neutrophil and monocyte function, impairs phagocytosis and leads to increased production of proinflammatory cytokines. ${ }^{16}$ The resulting immune-suppression may lead to increased tumour spread. So as anesthesiologist we should be cautious in the perioperative period and minimise such factors which may promote metastasis.

Another equally worrisome hypothesis which has been suggested is that the pro-tumor environment during anaesthesia for non-cancer surgery may activate dormant cancer cells in a patient where clinical malignancy would not have developed otherwise. ${ }^{21}$ Also though effective pain management is known to improve the quality of life of the cancer patient, but chronic opioid administration for pain management may also lead to immunosupression and subsequent metastasis and recurrence.

Opioids inhibit CTL and NK cell function and also increase angiogenesis which may promote tumor cell growth. ${ }^{22}$ Various clinical studies have shown that in clinically used doses opioids promotes endothelial cell proliferation and migration in vitro. Pro angiogenic effect of opioids ( $\mu$ receptor agonists) is due to reciprocal activation of VEGF (vascular endothelial growth factor). ${ }^{23}$ Further these $\mu$ receptor agonists also induce a defect in the barrier function 
of cells which allows penetration and subsequent metastasis of tumor cells. Morphine sensitive $\mu 3$-receptor on immune cells is responsible for its immunomodulation effects. ${ }^{24}$ So, use of peripherally acting $\mu$ receptor antagonists (like methyl-naltrexone) in the perioperative period, which do not antagonise their central analgesic effect, may be beneficial in attenuating pro-tumor effects of opioids. ${ }^{25}$ Synthetic opioids like fentanyl do not bind to $\mu 3$-receptor, so have less immunosuppressive effect than morphine. ${ }^{26}$ Fentanyl augments NK cell activity and increases the number of NK cells. ${ }^{27}$ Remifentanyl also does not impair NK activity. ${ }^{28}$ The therapeutic potential of peripheral opioid antagonist remains to be explored in future.

Volatile anaesthetics up-regulate the expression of hypoxia inducible factor- $1 \alpha$ (HIF-1 $\alpha)$, which is an important mediate for protection from ischemia. HIF system is also used by cancer cells to promote their own survival under condition of hypoxia. ${ }^{29}$ The level of HIF- $1 \alpha$ has been found to correlate with early relapse in breast cancer. Isoflurane is found to protect colon cancer cells from tumour necrosis factor (TNF) induced apoptosis. ${ }^{30}$ However, a recent study on sevoflurane failed to prove its association with increased risk of new cancer. ${ }^{31}$ For nitrous oxide the results of various studies have been conflicting. ${ }^{32}$ Certain anesthetic techniques and agents have been found to be associated with less immunosupression and cancer recurrence in cancer patients. Minor innocuous modifications in the anaesthetic management may thus lead to significant long term impact on outcome of onco-patients.

Regional anesthesia (RA) ameliorates most of the aforesaid adverse consequences of surgery in a cancer patient primarily by reducing the neuroendocrine stress response, requirement of inhalation agents and use of opioids in the perioperative period. ${ }^{33,34}$ Many large clinical trials have shown beneficial effect of RA to decrease the recurrence and improve survival, ${ }^{34-36}$ but others have refuted the claims. ${ }^{37,38}$ Benefit of epidural anaesthesia has been shown in trials that have tightly controlled pathological classification. ${ }^{39} \mathrm{RA}$ alone or supplemented with GA has well established role in improving quality of analgesia and decreasing postoperative morbidities. Thus, although not conclusively proven in reducing the incidence of recurrence of cancer, it thus seems justified to advocate its wider use in cancer surgery. Among the intravenous agents, midazolam ${ }^{40}$ and ketamine ${ }^{41}$ have been found to interfere with host immunity whereas propofol may have anti-tumour effect. Ketamine leads to profound immunosupression because of its adrenergic activity. Propofol attenuates surgical stress induced immunosupression and promotes T lymphocyte activities. ${ }^{42}$ Propofol also has cyclooxygenase (COX-2) inhibiting property. ${ }^{43}$ It has been found to inhibit lymphoma growth and the invasiveness of colon cancer cells. Thus, TIVA with propofol may be an attractive option for anaesthetising cancer patients.

COX-2 is over expressed in many types of cancers. It is critical for the production of prostaglandin PGE2 which promotes tumour progression. NSAIDS especially those with COX-2 selection inhibition, may prevent morphine induced tumour growth and metastasis since morphine stimulates COX-2 expression in tumour cells. ${ }^{44}$ Also, by reducing the requirement of opioids, they can minimize opioid induced immunosupression in the host. A clinical study showed that single dose of pre-emptive ketorolac significantly reduced recurrence after breast cancer surgery ${ }^{45}$ Other agents which may have a positive effect in preventing recurrence after cancer surgery are local anaesthetics ${ }^{46}$ and beta blockers. ${ }^{47}$ Local anaesthetics act by inhibiting voltage gated sodium channels (VGSC). These channels are highly expressed in a variety of cancer cells. These VGSCs are usually neonatal splice variants which are more sensitive to lidocaine and phenytoin than their adult counterparts. VGSC $\alpha$-subunit levels correlate with the metastatic potential of the tumours. In vitro exposure of human fibroblasts to local anaesthetics impairs cell growth. Mesenchymal stem cells may be critical in tumour growth and metastasis. Anti-proliferative effects of local anaesthetics may be mediated by potassium or calcium channels. Clinical evidence of the effectiveness of local anaesthetics to decrease tumour growth is however limited. Large randomised trials are required to further substantiate this effect.

Animal studies have shown that metastasis rate is halved with the administration of beta blockers. In humans, beneficial effects have been found in hypertensive patients on chronic beta blocker therapy with operable breast cancer in terms of longer disease free survival rates. They may exert their anti-tumour effect by reducing surgical stress response. Also, VEGF secretion is reduced from ovarian tumours following beta blockade. They may provide an exciting area for future research in cancer treatment. Other factors which may be of importance to a cancer patient in this regard are perioperative immunonutrition, prevention of inadvertent intraoperative hypoxia, hypothermia, hypotension and hyperglycemia. There is some evidence that perioperative immunonutrition with arginine and omega-3 fatty acids can improve NK cell activity and shift the TH1/Th2 balance in favour of Th1 and thus reduce immunosuppressant in cancer patients. ${ }^{48,49}$

\section{Conclusion}

Perioperative period is highly conducive for dissemination of cancer cells and later establishment of metastasis. The possibility of certain anaesthetic agents leading to increased recurrence after cancer surgery is a grave one and cannot be discounted. Though studies in this field are mostly inconclusive and limited by multiple confounding factors and mostly retrospective, enough experimental and clinical evidence has been generated to create a platform for future larger prospective randomised trials which might provide us with better clinical guide. Till then based on available evidence we can modify our anaesthetic practice for cancer patients to include safer techniques and anaesthetic agents to optimize outcomes. Regional anaesthesia and analgesia should be widely incorporated in the management of cancer patients.

\section{Funding details}

None.

\section{Acknowledgments}

None.

\section{Conflicts of interests}

Authors declare that there is no conflict of interest.

\section{References}

1. Camara O, Kavallaris A, Noschel H, et al. Seeding of epithelial cells into circulation during surgery for breast cancer:the fate of malignant and benign mobilized cells. World J Surg Oncol. 2006;4:67.

2. Eschwege P, Dumas F, Blanchet P, et al. Haematogenous dissemination of prostatic epithelial cells during radical prostatectomy. Lancet. 1995;346(8989):1528-1530.

3. Exadaktylos AK, Buggy DJ, Moriarty DC, et al. Can anesthetic technique for primary breast cancer surgery affect recurrence or metastasis? Anesthesiology. 2006;105(4):660-664. 
4. Gupta A, Bjornsson A, Fredriksson M, et al. Reduction in mortality after epidural anaesthesia and analgesia in patients undergoing rectal but not colonic cancer surgery:a retrospective analysis of data from 655 patients in Central Sweden. Br J Anaesth. 2011;107(2):164-170.

5. A Kelbel I, Weiss M. Anaesthetics and immune function. Curr Opin Anaesthesiol. 2011;14(6):685-691.

6. Schneemilch CE, Hachenberg $\mathrm{T}$, Ansorge S, et al. Effects of differentanaesthetic agents on immune cell function in vitro. Eur $J$ Anaesthesiol. 2005;22(8):616-623.

7. Wuethrich PY, Hsu Schmitz SF, Kessler TM, et al. Potential influence of the anesthetic technique used during open radical prostatectomy on prostate cancerrelated outcome:a retrospective study. Anesthesiology. 2010;113(3):570-576.

8. Kalluri R, Weinberg RA. The basics of epithelial-mesenchymal transition. J Clin Invest. 2009;119(6):1420-1428.

9. Joyce JA, Pollard JW. Microenvironmental regulation of metastasis. Nat Rev Cancer. 2009;9(4):239-252.

10. Polyak K, Weinberg RA. Transitions between epithelial and Mesenchymal states:acquisition of malignant and stem cell traits. Nat Rev Cancer. 2009;9(4):265-273.

11. Tsuchiya Y, Sawada S, Yoshioka I, et al. Increased surgical stress promotes tumor metastasis. Surgery. 2003;133(5):547-555.

12. Yang Q, Goding SR, Hokland ME, et al. Antitumor activity of NK cells. Immunol Res. 2006;36(1-3):13-25.

13. Zamai L, Ponti C, Mirandola P, et al. NK cells and cancer. J Immunol. 2007; 178:4011-4016.

14. Weber RS, Jabbour N, Martin RC. Anemia and transfusions in patients undergoing surgery for cancer. Ann Surg Oncol. 2008;15(1):34-45.

15. Ben-Eliyahu S, Shakhar G, Rosenne E, et al. Hypothermia in barbiturateanesthetized rats suppresses natural killer activity and compromises resistance to tumor metastasis. Anesthesiology. 1999;91(3):732-740.

16. Turina M, Fry DE, Polk HC. Acute hyperglycemia and innate immune system:clinical, cellular, and molecular aspects. Crit Care Med. 2005;33(7):1624-1633.

17. Van Meurs M, Wulfelt FM, Jongman RM, et al. Hemorrhagic shockinduced endothelial cell activation in a spontaneous breathing and a mechanical ventilation hemorrhagic shock model is induced by a proinflammatory response and not by hypoxia. Anesthesiology. 2011;115(3):474-482.

18. Eitzschig HK, Carmeliet P. Hypoxia and inflammation. $N$ Engl J Med. 2011;364(7):656-665

19. Cata JP, Wang H, Gottumukkala V, et al. Inflammatory response, immunosuppression, and cancer recurrence after perioperative blood transfusions. Br J Anaesth. 2013;110(5):690-701.

20. Lange MM, van Hilten JA, van de Watering LM, et al. Leucocyte depletion of perioperative blood transfusion does not affect long-term survival and recurrence in patients with gastrointestinal cancer. $\mathrm{Br} J$ Surg. 2009;96(7):734-740.

21. Bovill JG. Surgery for cancer:does anesthesia matter? Anesth Analg. 2010;110(6):1524-1526.

22. Sacerdote P. Opioid-induced immunosuppression. Curr Opin Support Palliat Care. 2008;2(1):14-18.

23. Chen C, Farooqui M, Gupta K. Morphine stimulates vascular endothelial growth factor-like signaling in mouse retinal endothelial cells. Curr Neurovasc Res. 2006;3(3):171-180.

24. Welters ID, Menzebach A, Goumon Y, et al. Morphine suppresses complement receptor expression, phagocytosis, and respiratory burst in neutrophils by a nitric oxide and $\mathrm{m} 3$ oipate receptor-dependent mechanism. J Neuroimmunol. 2000;111(1-2):139-145.
25. Singleton PA, Lingen MW, Fekete JG, et al. Methylnaltrexone inhibits opiate and VEGF-induced angiogenesis:role of receptor transactivation. Micrivasc Res. 2006;72(1-2):3-11.

26. Bilfinger TV, Fimiani C, Stefano GB. Morphines immunoregulatory actions are not shared by fentanyl. J Cardiol. 1998;64(Suppl 1):S61S66.

27. Yeager MP, Procopio MA, DeLeo JA, et al. Intravenous fentanyl increases natural killer cell cytotoxicity and circulating CD16p lymphocytes in human. Anesth Analg. 2002;94(1):94-99.

28. Cronin AJ, Aucutt-Walter NM, Budinetz T, et al. Low-dose remifentanil infusion does not impair natural killer function in healthy volunteers. $\mathrm{Br}$ J Anaesth. 2003;91(6):805-809.

29. Ma D, Lim T, Xu J, et al. Xenon preconditioning protects against renal ischemic-reperfusion injury via HIF-1alpha activation. $\mathrm{J} \mathrm{Am} \mathrm{Soc}$ Nephrol. 2009;20(4):713-720.

30. Wang C, Weihrauch D, Schwabe D, et al. Extracellular signal regulated kinases trigger isoflurane preconditioning concomitant with upregulation of hypoxia-inducible factor-1alpha and vascular endothelial growth factor expression in rats. Anesth Analg. 2006;103(2):281-288.

31. Lindholm M, Granath F, Eriksson L, et al. Malignant disease within 5 years after surgery in relation to duration of sevoflurane anesthesia and time with bispectral index under 45. Anesth Analg. 2011;113(4):778-783.

32. Fleischmann E, Marschalek C, Schlemitz K, et al. Nitrous oxide may not increase the risk of cancer recurrence after colorectal surgery:a followup of a randomized controlled trial. BMC Anesthesiol. 2009;9:1.

33. Kehlet H. Manipulation of the metabolic response in clinical practice. World J Surg. 2000;24(6):690-695.

34. Tonnesen E, Wahlgreen C. Influence of extradural and general anaesthesia on natural killer cell activity and lymphocyte subpopulations in patients undergoing hysterectomy. BrJ Anaesth. 1988;60(5):500-507.

35. Ahlers O, Nachtigall I, Lenze $J$, et al. Intraoperative thoracic epidural anaesthesia attenuates stress-induced immunosuppression in patients undergoing major abdominal surgery. $\mathrm{Br} J$ Anaesth. 2008;101(6):781-787.

36. Wada H, Seki S, Takahashi T, et al. Combined spinal and general anesthesia attenuates liver metastasis by preserving TH1/TH2 cytokine balance. Anesthesiology. 2007;106(3):499-506.

37. Myles PS, Peyton P, Silbert B, et al. Perioperative epidural analgesia for major abdominal surgery for cancer and recurrence-free survival:randomized trial. $\mathrm{Br}$ Med J. 2011;342:d1491.

38. Gupta A, Bjarnsson A, Fredriksson M, et al. Reduction in mortality after epidural anaesthesia and analgesia in patients undergoing rectal but not colonic cancer surgery:a retrospective analysis of data from 655 patients in central Sweden. Br J Anaesth. 2011;107(2):164-170.

39. de Oliveira GS, Ahmad S, Schink JC, et al. Intraoperative neuraxial anesthesia but not postoperative neuraxial analgesia is associated with increased relapse-free survival in ovarian cancer patients after primary cytoreductive surgery. Reg Anesth Pain Med. 2011;36(3):271-277.

40. Ohta N, Ohashi Y, Takayama C, et al. Midazolam suppresses maturation of murine dendritic cells and priming of lipopolysaccharide-induced $t$ helper 1-type immune response. Anesthesiology. 2011;114(2):355-362.

41. Ohta N, Ohashi Y, Fujino Y. Ketamine inhibits maturation of bone marrowderiveddendritic cells and priming of the Th1-type immune response. Anesth Analg. 2009;109(3):793-800.

42. Ren XF, Li WZ, Meng FY, et al. Differential effects of propofol and isoflurane on the activation of T-helper cells in lung cancer patients. Anaesthesia. 2010;65(5):478-482.

43. Inada T, Kubo K, Shingu K. Possible link between cyclooxygenaseinhibiting and antitumor properties of propofol. $J$ Anesth. 2011;25(4):569-575. 
44. Farooqui M, Li Y, Rogers T, et al. COX-2 inhibitor celecoxib prevent chronic morphine-induced promotion of angiogenesis, tumor growth, metastasis and mortality, without compromising analgesia. Br J Cancer. 2007;97(11):1523-1531.

45. Forget P, Vandenhende J, Berliere M, et al. Do intraoperative analgesics influence breast cancer recurrence after mastectomy? A retrospective analysis. Anesth Analg. 2010;110(6):1630-1635.

46. Fraser SP, Diss JK, Chioni AM, et al. Voltage-gated sodium channel expression and potentiation of human breast cancer metastasis. Clin Cancer Res. 2005;11(15):5381-5389.
47. Powe DG, Voss MJ, Zanker KS, et al. Beta-blocker drug therapy reduces secondary cancer formation in breast cancer and improves cancer specific survival. Oncotarget. 2010;1(7):628-638.

48. Suzuki D, Furukawa K, Kimura F, et al. Effects of perioperative immunonutrition on cell-mediated immunity, T helper type 1 (Th1)/ Th2 differentiation, and Th17 response after pancreaticoduodenectomy. Surgery. 2010;148(3):573-581.

49. Matsuda A, Furukawa K, Takahashi, et al. Preoperative oral immune enhancing nutritional supplementation corrects TH1/TH2 imbalance in patients undergoing elective surgery for colorectal cancer. Dis Colon Rectum. 2006;49(4):507-516. 